\title{
OSCILLATION CRITERIA FOR SECOND ORDER HALF-LINEAR DIFFERENTIAL EQUATIONS WITH DELAY
}

\author{
RADICA BOJIČIĆ ${ }^{1, \star}$, TANJA JOVANOVIĆ ${ }^{2}$ \\ ${ }^{1}$ Faculty of Ekonomy, University of Priština, Kosovska Mitrovica, Serbia \\ ${ }^{2}$ Faculty of Natural Sciences and Mathematics, University of Priština, Kosovska Mitrovica, Serbia
}

\begin{abstract}
The oscillation criteria of different types of differential equations are often the topic of numerous scientific papers, because their application in nuclear physics, fluid mechanics, relativistic mechanics, the study of chemical reactions in the system and in general are large in science. In this paper, the oscillation criteria using averaging functions of the half-linear differential equation are generalized to the half-linear differential equation with delay, under the appropriate assumptions for the delay function. Suitable examples illustrate the application of set oscillation criteria.
\end{abstract}

Keywords: Half-linear differential equations with delay, Oscillation criteria, Averaging function.

\section{INTRODUCTION}

Part of the qualitative analysis of differential equations is particularly intense in the last thirty years. During this time, new testing methods were developed and important and useful results were obtained. Probably the highest-studied differential equation of the second order is the Sturm-Liouville linear differential equation of the second order:

$(L)$

$$
\left[p(t) u^{\prime}(t)\right]^{\prime}+q(t) u(t)=0
$$

In the last decade of the last century, significant progress was made in determining the qualitative similarity of the solution of the equation $(L)$ and the second-order half-linear differential equation:

$$
\left[p(t) \Phi\left(u^{\prime}(t)\right)\right]^{\prime}+q(t) \Phi(u(t))=0
$$

where $q \in C\left(\left[t_{0}, \infty\right)\right), p \in C^{1}\left(\left[t_{0}, \infty\right) ;(0, \infty)\right), \Phi: \mathbb{R} \rightarrow \mathbb{R}$ defined by $\Phi(s):=|s|^{\alpha-1} s, \alpha>0$ is a constant. Especially it is necessary to point out the articles of Mirzov (Mirzov, 1976) and Elbert (Elbert, 1979), who first established that the equations $(L)$ and $(H L)$ have similar properties describing the character of the oscillations solutions.

Between the large number of oscillation criteria shown using averaging functions, it can be noted that as a weight function is the most frequently used or positive, continuous differentiable function $\rho$, such that $\rho^{\prime}$ is a nonnegative and decreasing function, or the function $(t-s)^{\alpha}$ for $\alpha$ is a natural or real number greater than the unit, or product of these functions. Articles Philos (Philos, 1989) and $\mathrm{Li}(\mathrm{Li}, 1995)$ on the oscillatory of a linear differential equation were given a positive answer to the question posed by the mathematicians who dealt with this problem - can a wider family of functions be used as a weight function?

Ten years later, J. Manojlović, who made an outstanding contribution to the whole theory of the oscillation of differential equations, announces the paper (Manojlović, 1999) in which one step further.

In the last years of the last century, the attention of the author has attracted a second-order differential equation known as halflinear differential equation with a delay of form:

$(R H L)$

$$
\left[p(t)\left|u^{\prime}(t)\right|^{\alpha-1} u^{\prime}(t)\right]^{\prime}+q(t)|u(\tau(t))|^{\alpha-1} u(\tau(t))=0
$$

where $\alpha>0$ is a constant, and functions $p(t), q(t), \tau(t)$ which satisfy the conditions:

$$
p \in C^{1}\left(\left[t_{0}, \infty\right) ;[0, \infty)\right), p^{\prime}(t) \geqslant 0, \quad \text { na }\left[\mathrm{t}_{0}, \infty\right) ;
$$

$$
q \in C\left(\left[t_{0}, \infty\right) ;[0, \infty)\right)
$$

$$
\lim _{t \rightarrow \infty} \int_{t_{0}}^{t} p^{-\frac{1}{\alpha}}(s) d s=\infty
$$

By studying these equations, led to the conclusion that there is a certain qualitative similarity of its solutions and solutions of the equation $(H L)$. The contribution to this study is given in the papers (Hsu \& Yeh, 1996), (Kusano \& Naito, 1997), (Kusano \& Wang, 1995), (El-Sheikh \& Sallam, 2000) and (Wang, 1997).

In this paper, the given oscillatory criteria using averaging functions $(H L)$ given in the paper (Manojlović, 1999), will be generalized to the equation $(R H L)$.

\section{MAIN RESULTS}

Given the differential equation $(R H L)$, in which the functions $p, q$ and $\tau$ satisfy the conditions $(a)-(d)$. 
Theorem 1. The equation $(R H L)$ is an oscillatory if exist constant $\lambda \in(0,1)$ and function $H \in \mathcal{H}^{+}(\mathcal{D})$ such that it is:

$$
\lim _{t \rightarrow \infty} \frac{1}{H\left(t, t_{0}\right)} \text {. }
$$

$$
\int_{t_{0}}^{t}\left[q(s) H(t, s)\left(\frac{\tau(s)}{s}\right)^{\alpha}-\frac{p(s) h^{\alpha+1}(t, s)}{\lambda(\alpha+1)^{\alpha+1} H^{\alpha}(t, s)}\right] d s=\infty
$$

where is

$$
h(t, s)=-\frac{\partial H(t, s)}{\partial s}
$$

Proof. Suppose the opposite, that there exists a nonoscillatory solution of $u(t)$ of equation (RHL). According to Lemma 1.1. from (Bojičić, 2015), there exists $T_{0} \geqslant t_{0}$ such that $u(t)>0, u(\tau(t))>$ $0, u^{\prime}(t)>0 \wedge u^{\prime \prime}(t) \leqslant 0$ for $\forall t \geqslant T_{0}$.

We define in $\left[T_{0}, \infty\right)$ function $w$ as:

$$
w(t)=\frac{p(t)\left(u^{\prime}(t)\right)^{\alpha}}{u^{\alpha}(t)} .
$$

From here we have:

$$
\begin{gathered}
w^{\prime}(t)=\frac{\left[p(t)\left(u^{\prime}(t)\right)^{\alpha}\right]^{\prime}}{u^{\alpha}(t)}-\frac{\alpha p(t)\left(u^{\prime}(t)\right)^{\alpha+1}}{u^{\alpha+1}(t)} \\
=\frac{-q(t)(u(\tau(t)))^{\alpha}}{u^{\alpha}(t)}-\alpha p(t)\left[\frac{w(t)}{p(t)}\right]^{\frac{\alpha+1}{\alpha}} .
\end{gathered}
$$

According to Lemma 1.2. (Bojičić, 2015), for every $\mu \in(0,1)$, we obtain

$$
\left[\frac{u(\tau(t))}{u(t)}\right]^{\alpha} \geqslant \lambda\left(\frac{\tau(t)}{t}\right)^{\alpha}, t \geqslant T_{0}
$$

where is $\lambda=\mu^{\alpha} \in(0,1)$. Hence, we have

$$
w^{\prime}(t) \leqslant-q(t) \lambda\left(\frac{\tau(t)}{t}\right)^{\alpha}-\alpha \frac{w^{\frac{\alpha+1}{\alpha}}(t)}{p^{\frac{1}{\alpha}}(t)}, t \geqslant T_{0} .
$$

If we multiply last inequality by $H(t, s)$, and integrate it from $T$ to $t$ for $T \geqslant T_{0}$, we get:

$$
\begin{gathered}
\int_{T}^{t} w^{\prime}(s) H(t, s) d s \leqslant \\
-\int_{T}^{t} \lambda q(s)\left(\frac{\tau(s)}{s}\right)^{\alpha} H(t, s) d s-\int_{T}^{t} \alpha \frac{w^{\frac{\alpha+1}{\alpha}}(s)}{p^{\frac{1}{\alpha}}(s)} H(t, s) d s .
\end{gathered}
$$

Using integration by parts, we have

$$
\begin{gathered}
\int_{T}^{t} w^{\prime}(s) H(t, s) d s=\left.w(s) H(t, s)\right|_{s=T} ^{s=t}-\int_{T}^{t} w(s) \frac{\partial H(t, s)}{\partial s} d s= \\
=-w(T) H(t, T)+\int_{T}^{t} w(s) h(t, s) d s,
\end{gathered}
$$

so that from equality (3) we obtain

$$
\lambda \int_{T}^{t} q(s)\left(\frac{\tau(s)}{s}\right)^{\alpha} H(t, s) d s \leqslant
$$

$$
\leqslant w(T) H(t, T)-\int_{T}^{t} w(s) h(t, s) d s-\alpha \int_{T}^{t} \frac{w^{\frac{\alpha+1}{\alpha}}(s)}{p^{\frac{1}{\alpha}}(s)} H(t, s) d s .
$$

If we use a inequality Hardly, Littewood \& Polya (Hardly et al., 1988) and put

$$
\begin{gathered}
X=[\alpha H(t, s)]^{\frac{\alpha}{\alpha+1}} \frac{w(s)}{p^{\frac{1}{\alpha+1}}(s)} \\
Y=\frac{\alpha^{\frac{\alpha}{\alpha+1}}}{(\alpha+1)^{\alpha}} \frac{p^{\frac{\alpha}{a+1}}(s) h^{\alpha}(t, s)}{H^{\frac{\alpha^{2}}{\alpha+1}}(t, s)} \\
\gamma=\frac{\alpha+1}{\alpha}=1+\frac{1}{\alpha}>1,
\end{gathered}
$$

we get

$w(s) h(t, s)-\frac{\alpha H(t, s) w^{\frac{\alpha+1}{\alpha}}(s)}{p^{\frac{1}{\alpha}}(s)} \leqslant \frac{p(s) h^{\alpha+1}(t, s)}{(\alpha+1)^{\alpha+1} H^{\alpha}(t, s)}, t \geqslant s \geqslant T_{0}$.

Therefore, for $t \geqslant T \geqslant T_{0}$ is valid inequality

$$
\begin{gathered}
\lambda \int_{T}^{t} q(s)\left(\frac{\tau(s)}{s}\right)^{\alpha} H(t, s) d s \leqslant \\
w(T) H(t, T)+\int_{T}^{t} \frac{p(s) h^{\alpha+1}(t, s)}{(\alpha+1)^{\alpha+1} H^{\alpha}(t, s)} d s .
\end{gathered}
$$

Since $H \in \mathcal{H}^{+}$, i.e. monotonically non-increasing by $s$, then for every $t \geqslant T_{0} \geqslant t_{0}$

$$
H\left(t, t_{0}\right) \geqslant H\left(t, T_{0}\right)
$$

Therefore, from (5) we obtain

$$
\begin{gathered}
\lambda \int_{T_{0}}^{t} q(s)\left(\frac{\tau(s)}{s}\right)^{\alpha} H(t, s) d s \leqslant \\
w\left(T_{0}\right) H\left(t, T_{0}\right)+\int_{T_{0}}^{t} \frac{p(s) h^{\alpha+1}(t, s)}{(\alpha+1)^{\alpha+1} H^{\alpha}(t, s)} d s \\
\leqslant w\left(T_{0}\right) H\left(t, t_{0}\right)+\int_{t_{0}}^{t} \frac{p(s) h^{\alpha+1}(t, s)}{(\alpha+1)^{\alpha+1} H^{\alpha}(t, s)} d s,
\end{gathered}
$$

whence we conclude

$$
\begin{gathered}
\lambda \int_{t_{0}}^{t} q(s)\left(\frac{\tau(s)}{s}\right)^{\alpha} H(t, s) d s= \\
\lambda \int_{t_{0}}^{T_{0}} q(s)\left(\frac{\tau(s)}{s}\right)^{\alpha} H(t, s) d s+\lambda \int_{T_{0}}^{t} q(s)\left(\frac{\tau(s)}{s}\right)^{\alpha} H(t, s) d s \\
\leqslant \lambda H\left(t, t_{0}\right) \int_{t_{0}}^{T_{0}} q(s)\left(\frac{\tau(s)}{s}\right)^{\alpha} d s+H\left(t, t_{0}\right)\left|w\left(T_{0}\right)\right|+ \\
\int_{t_{0}}^{t} \frac{p(s) h^{\alpha+1}(t, s)}{(\alpha+1)^{\alpha+1} H^{\alpha}(t, s)} d s, t \geqslant T_{0} .
\end{gathered}
$$


From the last inequality it is obvious that:

$$
\begin{aligned}
\lim _{t \rightarrow \infty} \sup \frac{1}{H\left(t, t_{0}\right)} & \int_{t_{0}}^{t}\left[q(s) H(t, s)\left(\frac{\tau(s)}{s}\right)^{\alpha}-\frac{p(s) h^{\alpha+1}(t, s)}{\lambda(\alpha+1)^{\alpha+1} H^{\alpha}(t, s)}\right] d s \\
& \leqslant \int_{t_{0}}^{T_{0}} q(s)\left(\frac{\tau(s)}{s}\right)^{\alpha} d s+\frac{w\left(T_{0}\right)}{\lambda} .
\end{aligned}
$$

According to the condition $\left(\mathbb{C}_{1}\right)$, we obtain a contradiction. Hence, the equation $(R H L)$ doesn't have nonoscillatory solutions, i.e. its equation is oscillatory.

Corollary 2. Equation (RHL) is oscillatory if exist function $H \in$ $\mathcal{H}^{+}(\mathcal{D})$ such that hold conditions:

$$
\lim _{t \rightarrow \infty} \sup \frac{1}{H\left(t, t_{0}\right)} \int_{t_{0}}^{t} \frac{p(s) h^{\alpha+1}(t, s)}{H^{\alpha}(t, s)} d s<\infty
$$

and

$$
\lim _{t \rightarrow \infty} \sup \frac{1}{H\left(t, t_{0}\right)} \int_{t_{0}}^{t} q(s)\left(\frac{\tau(s)}{s}\right)^{\alpha} H(t, s) d s=\infty .
$$

In order to illustrate the previously proven criteria, we consider the following example:

Example 3. Consider the differential equation:

$$
\left[t^{v}\left|u^{\prime}(t)\right|^{\alpha-1} u^{\prime}(t)\right]^{\prime}+t^{\mu}\left|u\left(\frac{t}{3}\right)\right|^{a-1} u\left(\frac{t}{3}\right)=0
$$

where is $v, \alpha, \mu$ are arbitrary constants that satisfy the conditions $\mu>0$ and $0 \leqslant v<\alpha \neq 2$. We check the conditions of Theorem 1:

$$
\begin{gathered}
p(t)=t^{v}>0, \forall t \geqslant t_{0}, v \geqslant 0 ; \\
p^{\prime}(t)=v t^{\nu-1} \geqslant 0 \text { jer je } v \geqslant 0 ; \\
\int_{t_{0}}^{t} \frac{d s}{p^{\frac{1}{\alpha}}(s)}=\int_{t_{0}}^{t} \frac{d s}{s^{\frac{v}{\alpha}}}=\left.\frac{1}{1-\frac{v}{\alpha}} s^{1-\frac{v}{\alpha}}\right|_{t_{0}} ^{t}=\left.\frac{\alpha}{\alpha-v} s^{1-\frac{v}{\alpha}}\right|_{t_{0}} ^{t} \underset{t \rightarrow \infty}{\longrightarrow} \infty
\end{gathered}
$$

Let $H(t, s)=(t-s)^{2}$ weight function. Then:

$$
h(t, s)=-\frac{\partial H(t, s)}{\partial s}=2(t-s) \geqslant 0, t \geqslant s \geqslant t_{0}
$$

It remains to be determined whether the condition $\left(\mathbb{C}_{1}\right)$ is valid:

$$
\begin{gathered}
\frac{1}{t^{2}} \int_{t_{0}}^{t}\left[q(s) H(t, s)\left(\frac{\tau(s)}{s}\right)^{\alpha}-\frac{p(s) h^{\alpha+1}(t, s)}{\lambda(\alpha+1)^{\alpha+1} H^{\alpha}(t, s)}\right] d s \\
=\frac{1}{t^{2}} \int_{t_{0}}^{t}\left[s^{\mu}(t-s)^{2} \frac{1}{3^{\alpha}}-\frac{s^{v} 2^{\alpha+1}(t-s)^{\alpha+1}}{\lambda(\alpha+1)^{\alpha+1}(t-s)^{2 \alpha}}\right] d s \\
=\frac{1}{3^{\alpha} t^{2}} \int_{t_{0}}^{t} s^{\mu}(t-s)^{2} d s-\frac{2^{\alpha+1}}{t^{2}} \int_{t_{0}}^{t} s^{v}(t-s)^{1-\alpha} d s
\end{gathered}
$$

$$
\begin{gathered}
\geqslant \frac{t_{0}^{\mu}}{3^{\alpha} t^{2}} \int_{t_{0}}^{t}\left(t-t_{0}\right)^{2} d s-\frac{2^{\alpha+1} t^{v}}{t^{2}} \int_{t_{0}}^{t} s^{v}(t-s)^{1-\alpha} d s \\
=\frac{t_{0}^{\mu}}{3^{\alpha+1} t^{2}}\left(t-t_{0}\right)^{3}-\frac{2^{\alpha+1}}{t^{2-\nu}} \frac{1}{2-\alpha}\left(t-t_{0}\right)^{2-\alpha} \\
=\frac{t_{0}^{\mu} t^{3}}{3^{\alpha+1}}\left(1-\frac{t_{0}}{t}\right)^{3}-\frac{2^{\alpha+1}}{2-\alpha} \frac{1}{t^{\alpha-v}}\left(1-\frac{t_{0}}{t}\right)^{2-\alpha} \underset{t \rightarrow \infty}{\longrightarrow} \infty,
\end{gathered}
$$

where, due to the arbitrariness of constants $\lambda \in(0,1)$ we take $\lambda=\left(\frac{1}{\alpha+1}\right)^{\alpha+1}$.

Consequently, condition $\left(\mathbb{C}_{1}\right)$ is satisfied, hence from here follows equation $\left(E_{1}\right)$ is oscillatory by Theorem 1 .

Theorem 4. Suppose there is a function $H \in \widetilde{\mathcal{H}}(D)$ such that the following condition is satisfied:

$$
\lim _{t \rightarrow \infty} \sup \frac{1}{H\left(t, t_{0}\right)} \int_{t_{0}}^{t} \frac{p(s) h^{\alpha+1}(t, s)}{H^{\alpha}(t, s)} d s<\infty .
$$

If exist constant $\lambda \in(0,1)$ and function $\varphi \in C\left(\left[t_{o}, \infty\right)\right)$ such that:

$$
\lim _{t \rightarrow \infty} \sup \frac{1}{H(t, T)} \text {. }
$$

$$
\int_{T}^{t}\left[q(s) H(t, s)\left(\frac{\tau(s)}{s}\right)^{\alpha}-\frac{p(s) h^{\alpha+1}(t, s)}{\lambda(\alpha+1)^{\alpha+1} H^{\alpha}(t, s)}\right] d s \geqslant \varphi(T)
$$

for every $T \geqslant t_{0}$ and

$$
\int_{t_{0}}^{\infty} \frac{\varphi_{+}^{\frac{\alpha+1}{\alpha}}(s)}{p^{\frac{1}{\alpha}}(s)} d s=\infty
$$

then equation $(R H L)$ is oscillatory.

Proof. We suppose that there exists a solution $u(t)$ of equation (RHL) such that $u(t)>0, t \geqslant T_{0}$. Defining the function $w(t)$ as in the proof of Theorem 1, we get (4) and (5), for every $t \geqslant T \geqslant T_{0}$ Then, for (5), we have

$$
\begin{gathered}
\varphi(T) \leqslant \lim _{t \rightarrow \infty} \sup \frac{1}{H(t, T)} . \\
\cdot \int_{T}^{t}\left[q(s) H(t, s)\left(\frac{\tau(s)}{s}\right)^{\alpha}-\frac{p(s) h^{\alpha+1}(t, s)}{\lambda(\alpha+1)^{\alpha+1} H^{\alpha}(t, s)}\right] d s \leqslant \frac{w(T)}{\lambda}
\end{gathered}
$$

Therefore, it is true that:

$$
\varphi(T) \leqslant \frac{w(T)}{\lambda}, \text { for every } T \geqslant T_{0} .
$$

Now, we can conclude:

$$
\lim _{t \rightarrow \infty} \sup \frac{1}{H\left(t, T_{0}\right)} \int_{T_{0}}^{t} q(s) H(t, s)\left(\frac{\tau(s)}{s}\right)^{\alpha} d s \geqslant \varphi\left(T_{0}\right) .
$$

We define functions

$$
F(t):=\frac{1}{\lambda H\left(t, T_{0}\right)} \int_{T_{0}}^{t} w(s) h(t, s) d s, t \geqslant T_{0}
$$


and

$$
G(t):=\frac{\alpha}{\lambda H\left(t, T_{0}\right)} \int_{T_{0}}^{t} \frac{w^{\frac{\alpha+1}{\alpha}}(s)}{p^{\frac{1}{\alpha}}(s)} H(t, s) d s, \quad t \geqslant T_{0} .
$$

Then, by (4) and (7) we see that

$$
\begin{gathered}
\liminf _{t \rightarrow \infty}[G(t)-F(t)] \leqslant \frac{w\left(T_{0}\right)}{\lambda}- \\
-\limsup _{t \rightarrow \infty} \frac{1}{H\left(t, T_{0}\right)} \int_{T_{0}}^{t} q(s) H(t, s)\left(\frac{\tau(s)}{s}\right)^{\alpha} d s \\
\leqslant \frac{w\left(T_{0}\right)}{\lambda}-\varphi\left(T_{0}\right)<\infty .
\end{gathered}
$$

It remains to be proved

$$
\int_{T_{0}}^{\infty} \frac{|w(s)|^{\frac{\alpha+1}{\alpha}}}{p^{\frac{1}{\alpha}}(s)} d s<\infty
$$

If we suppose that (9) fails, there exists $T_{1}>T_{0}$ such that

$$
\int_{T_{0}}^{t} \frac{|w(s)|^{\frac{\alpha+1}{\alpha}}}{p^{\frac{1}{\alpha}}(s)} d s \geqslant \frac{\mu}{\alpha \xi} \text { for } t \geqslant T_{1},
$$

where $\mu>0$ is arbitrary number, and $\xi$ is a positive constant, such that

$$
\inf _{s \geqslant T_{0}}\left(\liminf _{t \rightarrow \infty} \frac{H(t, s)}{H\left(t, t_{0}\right)}\right)>\xi>0 \text {. }
$$

Then we have

$$
\begin{aligned}
& G(t)=\frac{\alpha}{\lambda H\left(t, T_{0}\right)} \int_{T_{0}}^{t} \frac{w^{\frac{\alpha+1}{\alpha}}(s)}{p^{\frac{1}{\alpha}}(s)} H(t, s) d s= \\
& =\frac{\alpha}{\lambda H\left(t, T_{0}\right)} \int_{T_{0}}^{t} H(t, s) d\left(\int_{T_{0}}^{s} \frac{w^{\frac{\alpha+1}{\alpha}}(\delta)}{p^{\frac{1}{\alpha}}(\delta)} d \delta\right)= \\
& =\left.\frac{\alpha}{\lambda H\left(t, T_{0}\right)}\left(H(t, s) \int_{T_{0}}^{s} \frac{w^{\frac{\alpha+1}{\alpha}}(\delta)}{p^{\frac{1}{\alpha}}(\delta)} d \delta\right)\right|_{s=T_{0}} ^{s=t}- \\
& -\frac{\alpha}{\lambda H\left(t, T_{0}\right)} \int_{T_{0}}^{t} \frac{\partial H(t, s)}{\partial s}\left(\int_{T_{0}}^{s} \frac{w^{\frac{\alpha+1}{\alpha}}(\delta)}{p^{\frac{1}{\alpha}}(\delta)} d \delta\right) d s= \\
& =-\frac{\alpha}{\lambda H\left(t, T_{0}\right)} \int_{T_{0}}^{t} \frac{\partial H(t, s)}{\partial s}\left(\int_{T_{0}}^{s} \frac{w^{\frac{\alpha+1}{\alpha}}(\delta)}{p^{\frac{1}{\alpha}}(\delta)} d \delta\right) d s \\
& \geqslant-\frac{\alpha}{\lambda H\left(t, T_{0}\right)} \int_{T_{1}}^{t} \frac{\partial H(t, s)}{\partial s}\left(\int_{T_{0}}^{s} \frac{w^{\frac{\alpha+1}{\alpha}}(\delta)}{p^{\frac{1}{\alpha}}(\delta)} d \delta\right) d s \\
& \geqslant-\frac{\mu}{\lambda \xi H\left(t, T_{0}\right)} \int_{T_{1}}^{t} \frac{\partial H(t, s)}{\partial s} d s=-\left.\frac{\mu}{\lambda \xi H\left(t, T_{0}\right)} H(t, s)\right|_{s=T_{1}} ^{s=t}
\end{aligned}
$$

$$
=\frac{\mu}{\lambda \xi} \frac{H\left(t, T_{1}\right)}{H\left(t, T_{0}\right)}=\frac{\bar{\mu}}{\xi} \frac{H\left(t, T_{1}\right)}{H\left(t, T_{0}\right)}, \text { for all } t \geqslant T_{1}>T_{0},
$$

where with $\bar{\mu}$ we denote $\bar{\mu}=\frac{\mu}{\lambda}$. By (11) there is a $T_{2} \geqslant T_{1}$ such thate $\frac{H\left(t, T_{1}\right)}{H\left(t, T_{0}\right)} \geqslant \xi$ for all $t \geqslant T_{2}$, we conclude that

$$
G(t) \geqslant \bar{\mu} \text {, for all } t \geqslant T_{2} .
$$

Since $\bar{\mu}=\frac{\mu}{\lambda}$, and $\mu$ is arbitrary number, we get

$$
\lim _{t \rightarrow \infty} G(t)=\infty
$$

Consider now the number sequence $\left\{\sigma_{n}\right\}_{n=1}^{\infty}$ in $\left(T_{0}, \infty\right)$ such that

$$
\lim _{n \rightarrow \infty} \sigma_{n}=\infty
$$

and

$$
\lim _{n \rightarrow \infty}\left[G\left(\sigma_{n}\right)-F\left(\sigma_{n}\right)\right]=\liminf _{t \rightarrow \infty}[G(t)-F(t)]<\infty .
$$

Then, there exists a constant $M$ such that for all sufficiently large $n$ holds:

$$
G\left(\sigma_{n}\right)-F\left(\sigma_{n}\right) \leqslant M .
$$

Since (12) ensures that

$$
\lim _{n \rightarrow \infty} G\left(\sigma_{n}\right)=\infty,
$$

and (13) implies

$$
\lim _{n \rightarrow \infty} F\left(\sigma_{n}\right)=\infty .
$$

From equations (14) and (15), for sufficiently large $n$, we derive:

$$
\frac{F\left(\sigma_{n}\right)}{G\left(\sigma_{n}\right)}-1 \geqslant-\frac{M}{G\left(\sigma_{n}\right)}>-\frac{1}{2},
$$

i.e. $\frac{F\left(\sigma_{n}\right)}{G\left(\sigma_{n}\right)}>1$. Therefore, by using (15), we get:

$$
\lim _{n \rightarrow \infty} \frac{F^{\alpha+1}\left(\sigma_{n}\right)}{G^{\alpha}\left(\sigma_{n}\right)}=\infty .
$$

On the other hand, by Hölder's inequality, for every $n \in N$ we have

$$
\begin{aligned}
& F\left(\sigma_{n}\right)=\frac{1}{\lambda H\left(\sigma_{n}, T_{0}\right)} \int_{T_{0}}^{\sigma_{n}} w(s) h\left(\sigma_{n}, s\right) d s= \\
& =\frac{1}{\lambda} \int_{T_{0}}^{\sigma_{n}}\left(\frac{\alpha^{\frac{\alpha}{\alpha+1}}}{H^{\frac{\alpha}{\alpha+1}}\left(\sigma_{n}, T_{0}\right)} \frac{w(s) H^{\frac{\alpha}{\alpha+1}}\left(\sigma_{n}, s\right)}{p^{\frac{1}{\alpha+1}}(s)}\right) . \\
& \cdot\left(\frac{\alpha^{-\frac{\alpha}{\alpha+1}}}{H^{\frac{1}{\alpha+1}}\left(\sigma_{n}, T_{0}\right)} \frac{h\left(\sigma_{n}, s\right) p^{\frac{1}{\alpha+1}}(s)}{H^{\frac{\alpha}{\alpha+1}}\left(\sigma_{n}, s\right)}\right) d s \\
& \leqslant \frac{1}{\lambda}\left[\frac{\alpha}{H\left(\sigma_{n}, T_{0}\right)} \int_{T_{0}}^{\sigma_{n}} \frac{w^{\frac{\alpha+1}{\alpha}}(s) H\left(\sigma_{n}, s\right)}{p^{\frac{1}{\alpha}}(s)} d s\right]^{\frac{\alpha}{\alpha+1}} . \\
& \cdot\left[\frac{1}{\alpha^{\alpha} H\left(\sigma_{n}, T_{0}\right)} \int_{T_{0}}^{\sigma_{n}} \frac{p(s) h^{\alpha+1}\left(\sigma_{n}, s\right)}{H^{\alpha}\left(\sigma_{n}, s\right)} d s\right]^{\frac{1}{\alpha+1}} \\
& =\frac{1}{\lambda}\left(\frac{1}{\lambda}\right)^{-\frac{\alpha}{\alpha+1}} G^{\frac{\alpha}{\alpha+1}}\left(\sigma_{n}\right)\left[\frac{1}{\alpha^{\alpha} H\left(\sigma_{n}, T_{0}\right)} \int_{T_{0}}^{\sigma_{n}} \frac{p(s) h^{\alpha+1}\left(\sigma_{n}, s\right)}{H^{\alpha}\left(\sigma_{n}, s\right)} d s\right]^{\frac{1}{\alpha+1}},
\end{aligned}
$$


which gives

$$
\begin{gathered}
\frac{F^{\alpha+1}\left(\sigma_{n}\right)}{G^{\alpha}\left(\sigma_{n}\right)} \leqslant \frac{1}{\lambda \alpha^{\alpha} H\left(\sigma_{n}, T_{0}\right)} \int_{T_{0}}^{\sigma_{n}} \frac{p(s) h^{\alpha+1}\left(\sigma_{n}, s\right)}{H^{\alpha}\left(\sigma_{n}, s\right)} d s \\
\leqslant \frac{1}{\lambda \alpha^{\alpha} H\left(\sigma_{n}, t_{0}\right)} \int_{t_{0}}^{\sigma_{n}} \frac{p(s) h^{\alpha+1}\left(\sigma_{n}, s\right)}{H^{\alpha}\left(\sigma_{n}, s\right)} d s .
\end{gathered}
$$

So, because of (16), we have

$$
\lim _{n \rightarrow \infty} \frac{1}{H\left(\sigma_{n}, t_{0}\right)} \int_{t_{0}}^{\sigma_{n}} \frac{p(s) h^{\alpha+1}\left(\sigma_{n}, s\right)}{H^{\alpha}\left(\sigma_{n}, s\right)} d s=\infty
$$

i.e.

$$
\lim _{t \rightarrow \infty} \frac{1}{H\left(t, t_{0}\right)} \int_{t_{0}}^{t} \frac{p(s) h^{\alpha+1}(t, s)}{H^{\alpha}(t, s)} d s=\infty,
$$

and this is in contradiction with the condition $\left(\mathbb{C}_{2}\right)$. Therefore, (9) holds. Now, from (6), we obtain

$$
\int_{t_{0}}^{\infty} \frac{\varphi_{+}^{\frac{\alpha+1}{\alpha}}(s)}{p^{\frac{1}{\alpha}}(s)} d s \leqslant \int_{t_{0}}^{T_{0}} \frac{\varphi_{+}^{\frac{\alpha+1}{\alpha}}(s)}{p^{\frac{1}{\alpha}}(s)} d s+\lambda^{-\frac{\alpha+1}{\alpha}} \int_{T_{0}}^{\infty} \frac{w^{\frac{\alpha+1}{\alpha}}(s)}{p^{\frac{1}{\alpha}}(s)} d s<\infty
$$

which contradicts with the condition $\left(\mathbb{C}_{4}\right)$. This completes the proof.

Since Theorem 4 can be applied in certain cases where it is not possible to apply Theorem 1, the two oscillatory criteria are independent of each other. One such case is described in the following example.

Example 5. Consider the differential equation

$\left(E_{2}\right) \quad\left[t^{\nu}\left|u^{\prime}(t)\right|^{\alpha-1} u^{\prime}(t)\right]^{\prime}+\frac{(k+1)^{\alpha-1}}{t}\left|u\left(\frac{t}{3}\right)\right|^{\alpha-1} u\left(\frac{t}{3}\right)=0$

for $t \geqslant t_{0}$, where $k, v, \alpha$ are constants such that $k>0, v<\alpha, \alpha>2$.

If for the weight function we take the function $H(t, s)=$ $(t-s)^{2}$ and constant $\lambda=\left(\frac{1}{\alpha+1}\right)^{\alpha+1}$, we can determine that the condition $\left(\mathbb{C}_{1}\right)$ does not apply. Indeed, for $t \geqslant t_{0}$, we have:

$$
\begin{gathered}
\frac{1}{t^{2}} \int_{t_{0}}^{t}\left[q(s) H(t, s)\left(\frac{\tau(s)}{s}\right)^{\alpha}-\frac{p(\alpha) h^{\alpha+1}(t, s)}{\lambda(\alpha+1)^{\alpha+1} H^{\alpha}(t, s)}\right] d s \geqslant \\
\frac{1}{t^{2}} \int_{t_{0}}^{t}\left[\frac{(k+s)^{\alpha-1}}{s}(t-s)^{2}\left(\frac{1}{3}\right)^{\alpha}\right] d s-\frac{2^{\alpha+1}}{t^{2}} t^{\nu} \int_{t_{0}}^{t}(t-s)^{1-\alpha} d s \geqslant \\
\frac{1}{t^{2} 3^{\alpha}} \frac{\left(k+t_{0}\right)^{\alpha-1}}{t} \frac{\left(t-t_{0}\right)^{3}}{3}-\frac{2^{\alpha+1}}{t^{2}} t^{\nu} \frac{1}{2-\alpha}\left(t-t_{0}\right)^{2-\alpha} \geqslant \\
\frac{t_{0}^{\alpha-1}}{3^{\alpha+1}}\left(1-\frac{t_{0}}{t}\right)^{3}-\frac{2^{\alpha+1}}{(2-\alpha) t^{\alpha-1}}\left(1-\frac{t_{0}}{t}\right)^{2-\alpha} \underset{t \rightarrow \infty}{\longrightarrow} c=\text { const }<\infty .
\end{gathered}
$$

Hence, condition $\left(\mathbb{C}_{1}\right)$ is not satisfied, so we can not apply Theorem 1 . We check that the conditions $\left(\mathbb{C}_{2}\right),\left(\mathbb{C}_{3}\right)$ and $\left(\mathbb{C}_{4}\right)$ applies: $\left(\mathbb{C}_{2}\right)$

$$
\limsup _{t \rightarrow \infty} \frac{1}{t^{2}} \int_{t_{0}}^{t} \frac{p(s) h^{\alpha+1}(t, s)}{H^{\alpha}(t, s)} d s=\limsup _{t \rightarrow \infty} \frac{1}{t^{2}} \int_{t_{0}}^{t} s^{v}(t-s)^{1-\alpha} d s
$$

$$
\begin{gathered}
\leqslant \limsup _{t \rightarrow \infty} \frac{t^{\nu}}{t^{2}(2-\alpha)}\left(t-t_{0}\right)^{2-\alpha} \\
=\limsup _{t \rightarrow \infty} \frac{1}{2-\alpha} \frac{1}{t^{\alpha-v}}\left(1-\frac{t_{0}}{t}\right)^{2-\alpha}<\infty
\end{gathered}
$$

$\left(\mathbb{C}_{3}\right) \quad \frac{1}{t^{2}} \int_{T}^{t}\left[q(s) H(t, s)\left(\frac{\tau(s)}{s}\right)^{\alpha}-\frac{p(s) h^{\alpha+1}(t, s)}{\lambda(\alpha+1)^{\alpha+1} H^{\alpha}(t, s)}\right] d s$

$\geqslant \frac{T^{\alpha-1}}{3^{\alpha+1}}-\frac{2^{\alpha+1}}{(2-\alpha) t^{\alpha-\nu}}\left(1-\frac{T}{t}\right)^{2-\alpha} \geqslant \frac{T^{\alpha-1}}{3^{\alpha+1}}=\varphi(T)>0$

$\left(\mathbb{C}_{4}\right)$

$$
\begin{gathered}
\int_{t_{0}}^{\infty} \frac{\varphi^{\frac{\alpha+1}{\alpha}}(s)}{p^{\frac{1}{\alpha}}(s)} d s= \\
=\int_{t_{0}}^{\infty} \frac{s^{\frac{\alpha+1}{\alpha}(\alpha-1)} 3^{\frac{\alpha+1}{\alpha}(-1-\alpha)}}{s^{\frac{v}{\alpha}}} d s=\frac{1}{3^{\frac{(\alpha+1)^{2}}{\alpha}}} \int_{t_{0}}^{\infty} s^{\frac{\alpha^{2}-1}{\alpha}-\frac{v}{\alpha}} d s=\infty
\end{gathered}
$$

for $\alpha^{2}-1-v>0$, i.e. for $v<\alpha^{2}-1$. Accordingly, all conditions of Theorem 2 are satisfied, and hence, equation $\left(E_{2}\right)$ is oscillatory for $v<\min \left\{\alpha, \alpha^{2}-1\right\}$.

Theorem 6. Suppose that the function $H \in \widetilde{\mathcal{H}}(D)$ satisfies the condtion:

$\left(\mathbb{C}_{5}\right) \quad \limsup _{t \rightarrow \infty} \frac{1}{H\left(t, t_{0}\right)} \int_{t_{0}}^{t} q(s) H(t, s)\left(\frac{\tau(s)}{s}\right)^{\alpha} d s<\infty$.

If there exists constant $\lambda \in(0,1)$ and function $\varphi \in C\left(\left[t_{0}, \infty\right)\right)$ such that hold conditions $\left(\mathbb{C}_{4}\right)$ and

$$
\liminf _{t \rightarrow \infty} \frac{1}{H(t, T)} \text {. }
$$

$$
\cdot \int_{T}^{t}\left[q(s)\left(\frac{\tau(s)}{s}\right)^{\alpha} H(t, s)-\frac{p(s) h^{\alpha+1}(t, s)}{\lambda(\alpha+1)^{\alpha+1} H^{\alpha}(t, s)}\right] d s \geqslant \varphi(T)
$$

for all $T \geqslant t_{0}$, then equation (RHL) is oscillatory.

Proof. Suppose, on the contrary, that the equation $(R H L)$ has an nonoscillation solution $u(t)$. Without loss of generality, we assume that $u(t)>0$ for $t \geqslant T_{0}$. As in the proof of Theorem 1, holds (4) and (5) for all $t \geqslant T \geqslant T_{0}$. If we repeat the procedure as in the proof of Theorem 4 , using the condition $\left(\mathbb{C}_{6}\right)$, we obtain (6). Then from the condition $\left(\mathbb{C}_{5}\right)$ we have:

$$
\begin{aligned}
& \limsup _{t \rightarrow \infty}[G(t)-F(t)] \leqslant \frac{w\left(T_{0}\right)}{\lambda}-\liminf _{t \rightarrow \infty} \frac{1}{H\left(t, T_{0}\right)} . \\
& \int_{T_{0}}^{t} q(s)\left(\frac{\tau(s)}{s}\right)^{\alpha} H(t, s) d s \leqslant \frac{w\left(T_{0}\right)}{\lambda}-\varphi\left(T_{0}\right)<\infty
\end{aligned}
$$

By $\left(\mathbb{C}_{6}\right)$ we obtain

$$
\varphi\left(T_{0}\right) \leqslant \liminf _{t \rightarrow \infty} \frac{1}{H\left(t, T_{0}\right)} \int_{T_{0}}^{t} q(s)\left(\frac{\tau(s)}{s}\right)^{\alpha} H(t, s) d s-
$$




$$
-\liminf _{t \rightarrow \infty} \frac{1}{H\left(t, T_{0}\right)} \int_{T_{0}}^{t} \frac{p(s) h^{\alpha+1}(t, s)}{\lambda(\alpha+1)^{\alpha+1} H^{\alpha}(t, s)} d s,
$$

and by $\left(\mathbb{C}_{5}\right)$ we have:

$$
\liminf _{t \rightarrow \infty} \frac{1}{H\left(t, T_{0}\right)} \int_{T_{0}}^{t} \frac{p(s) h^{\alpha+1}(t, s)}{\lambda(\alpha+1)^{\alpha+1} H^{\alpha}(t, s)} d s<\infty
$$

If we form a number sequence $\left\{\sigma_{n}\right\}_{n=1}^{\infty}$ in $\left(T_{0}, \infty\right)$ that satisfies the conditions:

$$
\lim _{n \rightarrow \infty} \sigma_{n}=\infty
$$

and

$$
\lim _{n \rightarrow \infty}\left[G\left(\sigma_{n}\right)-F\left(\sigma_{n}\right)\right]=\limsup _{t \rightarrow \infty}[G(t)-F(t)]<\infty
$$

and apply the procedure as in the proof of Theorem 4, we can conclude that (9) holds, which with (6) again gives a contradiction to the condition $\left(\mathbb{C}_{4}\right)$.

If, apart from the parametric function $H(t, s)$ introduced as to weight function continuous differential function $\rho:\left[t_{0}, \infty\right] \rightarrow \mathbb{R}$, it can be shown the following oscillation criteria for equation $(R H L)$ :

Theorem 7. If exist constant $\lambda \in(0,1)$, positive, nondecreasing function $\rho \in C^{1}\left(\left[t_{0}, \infty\right)\right)$ and function $H \in \mathcal{H}^{+}(\mathbb{D})$, such that

$$
\begin{gathered}
\limsup _{t \rightarrow \infty} \frac{1}{H\left(t, t_{0}\right)} \cdot \\
\cdot \int_{t_{0}}^{t}\left[q(s)\left(\frac{\tau(s)}{s}\right)^{\alpha} H(t, s)-\frac{p(s) \rho(s) G^{\alpha+1}(t, s)}{\lambda(\alpha+1)^{\alpha+1} H^{\alpha}(t, s)}\right] d s=\infty
\end{gathered}
$$

where is

$$
G(t, s)=h(t, s)+\frac{\rho^{\prime}(s)}{\rho(s)} H(t, s)
$$

then equation $(R H L)$ is oscillatory.

Proof. Let $u(t)$ be a nonoscillatory solution of equation $(R H L)$. Without loss of generality, we assume that $u(t)>0$ for $t \geqslant t^{\prime}$. Then, according to Lemma 1.1. (Bojičić, 2015) $u^{\prime}(t)>0$ and $u^{\prime \prime}(t)<0$ for all $t \geqslant t_{0}$. Now, we define function

$$
W(t):=\rho(t) \frac{p(t)\left(u^{\prime}(t)\right)^{\alpha}}{u^{\alpha}(t)} \text { for } t \geqslant t_{0}
$$

Then, for every $t \geqslant t_{0}$

$$
\begin{aligned}
& W^{\prime}(t)=\rho^{\prime}(t) \frac{p(t)\left(u^{\prime}(t)\right)^{\alpha}}{u^{\alpha}(t)}+\rho(t) \frac{\left[p(t)\left(u^{\prime}(t)\right)^{\alpha}\right]^{\prime}}{u^{\alpha}(t)}- \\
&-\rho(t) \alpha p(t)\left(\frac{u^{\prime}(t)}{u(t)}\right)^{\alpha+1} .
\end{aligned}
$$

Therefore, according to Lemma 1.2. from (Bojičić, 2015), we obtain

$$
W^{\prime}(s) \leqslant W(s) \frac{\rho^{\prime}(s)}{\rho(s)}-\rho(s) q(s) \lambda\left(\frac{\tau(s)}{s}\right)^{\alpha}-\alpha \frac{W^{\frac{\alpha+1}{\alpha}}(s)}{(\rho(s) p(s))^{\frac{1}{\alpha}}}
$$

za for all $t \geqslant t_{0}=t_{0}(\lambda)$.
If we multiply (17) by $H(t, s)$ for $t \geqslant s \geqslant t_{0}$, integrate from $t_{0}$ to $t$, we get

$$
\begin{gathered}
\int_{t_{0}}^{t} W^{\prime}(s) H(t, s) d s \leqslant \\
\int_{t_{0}}^{t} W(s) \frac{\rho^{\prime}(s)}{\rho(s)} H(t, s) d s-\lambda \int_{t_{0}}^{t} \rho(s) q(s)\left(\frac{\tau(s)}{s}\right)^{\alpha} H(t, s) d s- \\
-\int_{t_{0}}^{t} \alpha \frac{W^{\frac{\alpha+1}{\alpha}}(s)}{(\rho(s) p(s))^{\frac{1}{\alpha}}} H(t, s) d s,
\end{gathered}
$$

i.e.

$$
\begin{gathered}
-W\left(t_{0}\right) H\left(t, t_{0}\right)-\int_{t_{0}}^{t} W(s) \frac{\partial H(t, s)}{\partial s} d s \leqslant \\
\int_{t_{0}}^{t} \frac{\rho^{\prime}(s)}{\rho(s)} W(s) H(t, s) d s-\lambda \int_{t_{0}}^{t} \rho(s) q(s)\left(\frac{\tau(s)}{s}\right)^{\alpha} H(t, s) d s- \\
-\int_{t_{0}}^{t} \alpha \frac{W^{\frac{\alpha+1}{\alpha}}(s)}{(\rho(s) p(s))^{\frac{1}{\alpha}}} H(t, s) d s .
\end{gathered}
$$

From this we conclude:

$$
\lambda \int_{t_{0}}^{t} \rho(s) q(s)\left(\frac{\tau(s)}{s}\right)^{\alpha} H(t, s) d s \leqslant
$$

$W\left(t_{0}\right) H\left(t, t_{0}\right)+\int_{t_{0}}^{t} W(s) G(t, s) d s-\alpha \int_{t_{0}}^{t} \frac{W^{\frac{\alpha+1}{\alpha}}(s)}{(\rho(s) p(s))^{\frac{1}{\alpha}}} H(t, s) d s$.

If, again, we use inequality Hardly, Littewood \& Polya and put:

$$
\begin{gathered}
X=(\alpha H(t, s))^{\frac{\alpha}{\alpha+1}} \frac{W(s)}{(\rho(s) p(s))^{\frac{1}{\alpha+1}}}, \\
Y=\left(\frac{\alpha}{\alpha+1}\right)^{\alpha}\left(\frac{p(s) \rho(s)}{(\alpha H(t, s))^{\alpha}}\right)^{\frac{\alpha}{\alpha+1}} G^{\alpha}(t, s), \\
\gamma=\frac{\alpha+1}{\alpha}
\end{gathered}
$$

we get that for all $t>s \geqslant t_{0}$ holds

$$
\alpha H(t, s) \frac{W^{\frac{\alpha+1}{\alpha}}(s)}{(\rho(s) p(s))^{\frac{1}{\alpha}}}+\frac{1}{(\alpha+1)^{\alpha+1}} \frac{p(s) \rho(s)}{H^{\alpha}(t, s)} G^{\alpha+1}(t, s)-
$$

$$
-W(s) G(t, s) \geqslant 0,
$$

which implies

$$
\begin{gathered}
W(s) G(t, s)-\alpha H(t, s) \frac{W^{\frac{\alpha+1}{\alpha}}(s)}{(\rho(s) p(s))^{\frac{1}{\alpha}}} \leqslant \\
\leqslant \frac{p(s) \rho(s)}{(\alpha+1)^{\alpha+1} H^{\alpha}(t, s)} G^{\alpha+1}(t, s) .
\end{gathered}
$$

Now, from (18) we get

$$
\lambda \int_{t_{0}}^{t} \rho(s) q(s)\left(\frac{\tau(s)}{s}\right)^{\alpha} H(t, s) d s \leqslant W\left(t_{0}\right) H\left(t, t_{0}\right)+
$$




$$
+\int_{t_{0}}^{t} \frac{p(s) \rho(s)}{(\alpha+1)^{\alpha+1} H^{\alpha}(t, s)} G^{\alpha+1}(t, s) d s
$$

so it is

$$
\limsup _{t \rightarrow \infty} \frac{1}{H\left(t, t_{0}\right)}
$$

$$
\begin{gathered}
\int_{t_{0}}^{t}\left[\rho(s) q(s)\left(\frac{\tau(s)}{s}\right)^{\alpha} H(t, s)-\frac{p(s) \rho(s)}{(\alpha+1)^{\alpha+1} H^{\alpha}(t, s)} G^{\alpha+1}(t, s)\right] d s \\
\leqslant \frac{W\left(t_{0}\right)}{\lambda}
\end{gathered}
$$

which contradicts $\left(\mathbb{C}_{7}\right)$.

Corollary 8. If exist constant $\lambda \in(0,1)$, positive, nondecreasing function $\rho \in C^{1}\left(\left[t_{0}, \infty\right)\right)$ and function $H \in \mathcal{H}^{+}(\mathbb{D})$, such that

$\left(\mathbb{C}_{8}\right) \quad \limsup _{t \rightarrow \infty} \frac{1}{H\left(t, t_{0}\right)} \int_{t_{0}}^{t} \frac{p(s) \rho(s)}{H^{\alpha}(t, s)} G^{\alpha+1}(t, s) d s<\infty$

and

$\left(\mathbb{C}_{9}\right) \quad \limsup _{t \rightarrow \infty} \frac{1}{H\left(t, t_{0}\right)} \int_{t_{0}}^{t} \rho(s) q(s)\left(\frac{\tau(s)}{s}\right)^{\alpha} H(t, s) d s=\infty$

then equation $(R H L)$ is oscillatory.

The purpose of introducing weighting function $\rho \in$ $C^{1}\left(\left[t_{0}, \infty\right)\right)$ is justified by the following example:

Example 9. Consider the differential equation

$$
\left[t^{v}\left|u^{\prime}(t)\right|^{\alpha-1} u^{\prime}(t)\right]^{\prime}+\frac{1}{t^{2}}\left|u\left(\frac{t}{3}\right)\right|^{\alpha-1} u\left(\frac{t}{3}\right)=0
$$

where is $v$ i $\alpha$ constants such that $0 \leqslant v<\alpha \neq 2$. If we take that functions $H(t, s)=(t-s)^{2}$ and $\rho(t)=t^{3}$, we can prove that the conditions $\left(\mathbb{C}_{8}\right)$ i $\left(\mathbb{C}_{9}\right)$ hold. Indeed:

$$
\begin{gathered}
\frac{1}{t^{2}} \int_{t_{0}}^{t} \frac{p(s) \rho(s)}{H^{\alpha}(t, s)} G^{\alpha+1}(t, s) d s= \\
=\frac{1}{t^{2}} \int_{t_{0}}^{t} \frac{s^{v+3}}{(t-s)^{2 \alpha}}\left[2(t-s)+\frac{3}{s}(t-s)^{2}\right]^{\alpha+1} d s= \\
=\frac{1}{t^{2}} \int_{t_{0}}^{t} s^{v+3}(t-s)^{1-\alpha} \frac{2^{\alpha+1}}{s^{\alpha+1}}[s+3(t-s)]^{\alpha+1} d s \leqslant \\
\leqslant \frac{2^{\alpha+1}}{t^{2}}\left(t+3\left(t-t_{0}\right)\right)^{\alpha+1}\left(t-t_{0}\right)^{1-\alpha} \frac{t^{\nu+2-\alpha+1}-t_{0}^{\nu+2-\alpha+1}}{v+2-\alpha+1} \leqslant \\
\leqslant 2^{\alpha+1} 4^{\alpha+1} t^{\alpha+1+1-\alpha-2} \frac{t^{\nu-\alpha+3}}{v-\alpha+3}= \\
=\frac{8^{\alpha+1}}{v-\alpha+3} t^{\nu-\alpha+3} \frac{\longrightarrow}{t \rightarrow \infty},
\end{gathered}
$$

when is $v-\alpha+3<0$, i.e. where $v<\alpha-3$ and

$$
\begin{gathered}
\text { (C) } \frac{1}{t^{2}} \int_{t_{0}}^{t} q(s)\left(\frac{\tau(s)}{s}\right)^{\alpha} \rho(s) H(t, s) d s=\frac{1}{t^{2}} \int_{t_{0}}^{t} \frac{s}{3^{\alpha}}(t-s)^{2} d s \geq \\
\geq \frac{t_{0}}{3^{\alpha+1} t^{2}}\left(t-t_{0}\right)^{3}=\frac{t_{0} t^{3}}{3^{\alpha+1}}\left(1-\frac{t_{0}}{t}\right)^{3} \underset{t \rightarrow \infty}{\longrightarrow} \infty .
\end{gathered}
$$

Hence, according to Corollary 5 , equation $\left(E_{3}\right)$ is oscillatory.

Using a Riccati's technique and starting from the generality of Riccati's differential inequality (17), i.e. the corresponding integral inequality (18), procedure as in the proof of Theorem 4 , i.e. Theorem 6 , can be prove the following two oscillation criteria for equation $(R H L)$ :

Theorem 10. Suppose that from $H \in \widetilde{\mathcal{H}}(D)$ hold

$$
\limsup _{t \rightarrow \infty} \frac{1}{H\left(t, t_{0}\right)} \int_{t_{0}}^{t} \frac{p(s) \rho(s)}{H^{\alpha}(t, s)} G^{\alpha+1}(t, s) d s<\infty .
$$

If there is function $\varphi \in C\left(\left[t_{0}, \infty\right)\right.$ which satisfies the condition $\left(\mathbb{C}_{4}\right)$ and for some constant $\lambda \in(0,1)$ condition

$$
\begin{gathered}
\limsup _{t \rightarrow \infty} \frac{1}{H(t, T)} . \\
\int_{T}^{t}\left[\rho(s) q(s)\left(\frac{\tau(s)}{s}\right)^{\alpha} H(t, s)-\frac{p(s) \rho(s)}{\lambda(\alpha+1)^{\alpha+1} H^{\alpha}(t, s)} G^{\alpha+1}(t, s)\right] d s \\
\geqslant \varphi(T)
\end{gathered}
$$

holds for all $T \geqslant t_{0}$, then equation (RHL) is oscillatory.

Theorem 11. Suppose that for fuction $H \in \tilde{\mathcal{H}}(D)$ holds

$$
\limsup _{t \rightarrow \infty} \frac{1}{H\left(t, t_{0}\right)} \int_{t_{0}}^{t} \rho(s) q(s)\left(\frac{\tau(s)}{s}\right)^{\alpha} H(t, s) d s<\infty .
$$

If there is function $\varphi \in C\left(\left[t_{0}, \infty\right)\right.$ which satisfies the condition $\left(\mathbb{C}_{4}\right)$ and for some constant $\lambda \in(0,1)$ condition

$$
\begin{gathered}
\limsup _{t \rightarrow \infty} \frac{1}{H(t, T)} . \\
\int_{T}^{t}\left[\rho(s) q(s)\left(\frac{\tau(s)}{s}\right)^{\alpha} H(t, s)-\frac{p(s) \rho(s)}{\lambda(\alpha+1)^{\alpha+1} H^{\alpha}(t, s)} G^{\alpha+1}(t, s)\right] d s \\
\geqslant \varphi(T)
\end{gathered}
$$

for all $T \geqslant t_{0}$, then equation $(R H L)$ is oscillatory.

Consider the differential equation $\left(R H L^{\prime}\right)$

$$
\left[p(t)\left|u^{\prime}(\theta(t))\right|^{\alpha-1} u^{\prime}(\theta(t))\right]^{\prime}+q(t)|u(\tau(t))|^{\alpha-1} u(\tau(t))=0
$$

where is functions $p, q, \tau$ satisfies conditions $(a)-(d)$ and function $\theta$ satisfy conditions

(e)

$\theta \in C^{1}\left(\left[t_{0}, \infty\right) ;[0, \infty)\right), \lim _{t \rightarrow \infty} \theta(t)=\infty, \theta^{\prime}(t) \geqslant 0$ for all $t \geqslant t_{0}$ 
and

$$
\theta(t) \geqslant t, \quad t \geqslant t_{0} .
$$

Using Lemma 1.1. from (Bojičić, 2015), if $u(t)>0, t \geqslant t_{0}$ is nonoscillatory solution of equation $\left(R H L^{\prime}\right)$ such that $u(\tau(t)) \mathrm{i}$ $u(\theta(t))>0, t \geqslant t_{0}$, then is $u^{\prime}(t)>0, u^{\prime}(\theta(t))>0, u^{\prime \prime}(t)<0 \mathrm{i}$ $u^{\prime \prime}(\theta(t))<0$ for $t \geqslant t_{0}$. For the function $w(t)$ defined by

$$
w(t)=\frac{p(t)\left(u^{\prime}(\theta(t))\right)^{\alpha}}{u^{\alpha}(t)}, t \geqslant T_{0}
$$

using by Lemma 1.2. from (Bojičić, 2015) we obtain

$$
\begin{gathered}
w^{\prime}(t)=-q(t) \frac{u^{\alpha}(\tau(t))}{u^{\alpha}(t)}-\alpha p(t) \frac{\left(u^{\prime}(\theta(t))\right)^{\alpha} u^{\prime}(t)}{u^{\alpha+1}(t)} \\
\leqslant-\lambda q(t)\left(\frac{\tau(t)}{t}\right)^{\alpha}-\alpha p(t)\left(\frac{u^{\prime}(\theta(t))}{u(t)}\right)^{\alpha+1}, t \geqslant T_{0}=T_{0}(\lambda) \geqslant t_{0} .
\end{gathered}
$$

Hence, for equation $\left(R H L^{\prime}\right)$ is valid Riccatie's inequality

$$
w^{\prime}(t) \leqslant-\lambda q(t)\left(\frac{\tau(t)}{t}\right)^{\alpha}-\alpha \frac{w^{\frac{\alpha+1}{\alpha}}(t)}{p^{\frac{1}{\alpha}}(t)}, \quad t \geqslant T_{0},
$$

which has the same shape as (2). We conclude that for equation $\left(R H L^{\prime}\right)$, all the above proven results will be valid.

\section{REFERENCES}

Bojičić, R. 2015. Oscillation properties of half-linear differential equation with delay. In Proceedings of the Sixth Mathematical Conference of the Republic of Srpska. in Serbian, pp. 122-134.

El-Sheikh, M. \& Sallam, R. 2000. Oscillation criteria for second order functional differential equations. Applied Mathematics and Computation, 115(2-3), pp. 113-121.

Elbert, A. 1979. A half-linear second order differential equation. Colloquia Mathematica Societatis Janos Bolyai, 30, pp. 153180.
Hardly, G., Littlewood, J., \& Polya, G. 1988. Inequalities. Cambridge University.

Hsu, H. \& Yeh, C. 1996. Oscillations theorems for second-order half-linear differential equations. Applied Mathematics Letters, 9(6), pp. 71-77.

Kusano, T. \& Naito, Y. 1997. Oscillation and nonoscillation criteria for second-order quasilinear differential equations. Mathematica Hungarica, 76(1-2), pp. 81-99.

Kusano, T. \& Wang, J. 1995. Oscillation properties of halflinear functional differential equation of the second order. Hiroshima Mathematical Journal, 25(2), pp. 371-385.

Li, H. J. 1995. Oscillation Criteria for Second Order Linear Differential Equations. Journal of Mathematical Analysis and Applications, 194(1), pp. 217-234. doi:10.1006/jmaa.1995.1295.

Manojlović, J. 1999. Oscillation criteria for second-order half-linear differential equations. Mathematical and Computer Modelling, 30(5-6), pp. 109-119. doi:10.1016/s08957177(99)00151-X.

Mirzov, J. D. 1976. On some analogs of Sturm's and Kneser's theorems for nonlinear systems. Journal of Mathematical Analysis and Applications, 53(2), pp. 418-425. doi:10.1016/0022$247 \times(76) 90120-7$.

Philos, C. G. 1989. Oscillation theorems for linear differential equations of second order. Archiv der Mathematik, 53(5), pp. 482-492. doi:10.1007/bf01324723.

Wang, J. 1997. Oscillation and nonoscillation theorems for a class of second order quasilinear functional-differential equations. Hiroshima Mathematical Journal, 27(3), pp. 449-466. doi:10.32917/hmj/1206126963. 